\title{
Switchable cell trapping using superparamagnetic beads
}

\author{
Matthew T. Bryan, ${ }^{1}$ Katherine H. Smith, ${ }^{2}$ Maria E. Real, ${ }^{2}$ M. A. Bashir, ${ }^{1}$ Paul W. Fry, ${ }^{3}$ \\ Peter Fischer, ${ }^{4}$ Mi-Young Im, ${ }^{4}$ Thomas Schrefl, ${ }^{1,5}$ Dan A. Allwood, ${ }^{1}$ John W. Haycock ${ }^{2}$ \\ ${ }^{1}$ Department of Engineering Materials, University of Sheffield, Sheffield, UK \\ ${ }^{2}$ The Kroto Research Institute, University of Sheffield, Sheffield, UK \\ ${ }^{3}$ Centre for Nanoscience and Technology, University of Sheffield, Sheffield, UK \\ ${ }^{4}$ Center for X-ray Optics, Lawrence Berkeley National Laboratory, Berkeley, California, USA \\ ${ }^{5}$ St. Poelten University of Applied Sciences, St. Poelten, Austria
}

\begin{abstract}
Ni}_{81} \mathrm{Fe}_{19}$ microwires are investigated as the basis of a switchable template for positioning magnetically-labeled neural Schwann cells. Magnetic transmission X-ray microscopy and micromagnetic modeling show that magnetic domain walls can be created or removed in zigzagged structures by an applied magnetic field. Schwann cells containing superparamagnetic beads are trapped by the field emanating from the domain walls. The design allows Schwann cells to be organized on a surface to form a connected network and then released from the surface if required. As aligned Schwann cells can guide nerve regeneration, this technique is of value for developing glial-neuronal co-culture models in the future treatment of peripheral nerve injuries.
\end{abstract}

\section{INTRODUCTION}

Superparamagnetic beads and wires are widely used within bioscience to separate, organize and manipulate biomolecules and cells [Tanase 2005, Inglis 2006, Desai 2007, Mirowski 2007, Vieira 2009]. The beads can be incorporated into biological cells without affecting cellular viability [Desai 2007] and are attracted towards magnetic poles [Mirowski 2004, Tanase 2005, Mirowski 2007, Conroy 2008, Vieira 2009]. Lithographed micrometer-scale magnetic structures enable the position of magnetic poles to be controlled, providing a method of influencing the absolute position of the beads on a surface. Cellular organization is attracting interest as a means of treating peripheral nerve injuries, since aligned Schwann cells are one requirement for the accurate guidance of regenerating neurites following injury [Sun 2008]. Here, cells are organized ex-vivo on a magnetic template. The method could be used on any cell type to study how cellular organization affects cell interactions in co-culture models.

The force on a superparamagnetic bead below magnetic saturation can be calculated from the gradient of the magnetic potential energy of the bead. This can be achieved by subdividing the bead into $N$ elements of equal volume. The total force, $F$, is then given by

$$
\vec{F}=\sum_{i}^{N} \frac{2 V \chi}{\mu_{0} N\left(1+N_{d} \chi\right)}\left(\vec{B}_{i} \cdot \vec{\nabla}\right) \vec{B}_{i}
$$

where $V$ is the volume of the bead, $\chi$ is the magnetic susceptibility of the bead, $\mu_{0}$ is the permeability of free space $N_{d}=1 / 3$ is the demagnetization factor of a sphere and $B_{i}$ is the magnetic induction at the $i^{\text {th }}$ element. Therefore, superparamagnetic beads are attracted towards points where both the field and the field gradient are large. The large field gradients that occur at the edges of lithographed magnetic structures can be utilized to trap, rotate or even translate the beads across a surface under various field conditions [Mirowski 2007, Conroy 2008]. As the beads may be attached to DNA strands or incorporated into cells [Tanase 2005, Mirowski 2007, Vieira 2009], this provides a mechanism of manipulating biological material. Recent investigations have demonstrated bead trapping or release depending on the presence or absence of a field [Mirowski 2004, Vieira 2009].

The magnetization of lithographed wires tends to lie along the long axis, with closure domains carrying a magnetic charge at the ends. Domain walls also carry a net magnetic charge, but unlike closure domains move under a magnetic field. Although domain walls in lithographed structures have generated a lot of interest in recent years for information technology applications [Allwood 2005, Parkin 2008], their use as a magnetic field source has received only limited attention [Allwood 2006, Vavassori 2009, Vieira 2009]. Here, micromagnetic modeling is used to compare the trapping potential of closure domains and domain walls. Structures containing domain walls are demonstrated to not only trap and align Schwann cells containing superparamagnetic beads, but also controllably release the cells. Our design creates a nonvolatile trapping state (ON or OFF) that remains after the field is removed, simplifying the experimental requirements for switchable traps.

\section{MODELING AND EXPERIMENTAL METHODS}

The Landau-Liftshitz-Gilbert equation was solved, using a hybrid finite element/boundary element micromagnetic model [Fredkin 1990] to find the relaxed magnetization state of a single planar microwire (SMW), two perpendicular microwires (PMW) and a curved microwire (CMW). The lateral dimensions of the structures are shown in Fig. 1a-c. SMW and 
PMW were $30 \mathrm{~nm}$ thick, CMW was $60 \mathrm{~nm}$ thick. All structures were meshed using a tetrahedral mesh. Due to restrictions from the structure size each structure has a unique cell size. A uniform $15 \mathrm{~nm} \times 15 \mathrm{~nm} \times 30 \mathrm{~nm}$ mesh was used for SMW, whereas a graded mesh was used for PMW and CMW, using a fine (coarse) mesh cell size of $10 \mathrm{~nm} \times 10 \mathrm{~nm} \times 30 \mathrm{~nm}$ (30 $\mathrm{nm} \times 30 \mathrm{~nm} \times 30 \mathrm{~nm})$ and $10 \mathrm{~nm} \times 10 \mathrm{~nm} \times 60 \mathrm{~nm}(30 \mathrm{~nm} \times 30$ $\mathrm{nm} \times 60 \mathrm{~nm}$ ), respectively. The lighter areas in figs. $1 \mathrm{~b}-\mathrm{c}$ indicate the finely meshed regions. The bulk material parameters for Permalloy $\left(\mathrm{Ni}_{80} \mathrm{Fe}_{20}\right)$ were used (exchange constant, $A=13 \mathrm{pJm}^{-1}$, saturation magnetization $M_{\mathrm{s}}=800$ $\mathrm{kAm}^{-1}$, magneto-crystalline anisotropy constant $K=0 \mathrm{Jm}^{-3}$ and damping coefficient $\alpha=0.01$ ) except for CMW, where $A$ was increased to $39 \mathrm{pJm}^{-1}$ in the finely meshed region $(A=390$ $\mathrm{pJm}^{-1}$ elsewhere). Lower $A$ in $\mathrm{CMW}$ resulted in a poor agreement with the experimental data, possibly because the mesh cells are larger than the exchange length of Permalloy. The stray field above the structures were calculated using a boundary element technique [Schrefl 2004] with $100 \mathrm{~nm} \times 100$ $\mathrm{nm} \times 50 \mathrm{~nm}$ cubic cells. Using (1), the force on a superparamagnetic bead $15 \mathrm{~nm}$ above each structure was calculated for a spherical shell of radius $200 \mathrm{~nm}$ and thickness $150 \mathrm{~nm}$ with $\chi=3.1$, similar to the experimental bead. This simulates the bead resting on a $15 \mathrm{~nm}$ thick tetraglyme layer used in the experiment.

Following the modeling, $1 \mu \mathrm{m}$ wide, $30 \mathrm{~nm}$ thick microwires similar to SMW and PMW and $2 \mu \mathrm{m}$ wide, $60 \mathrm{~nm}$ thick zigzagged microwires based on CMW were fabricated via electron beam lithography, followed by thermal evaporation of Permalloy and lift-off. Fig. 1d shows a confocal micrograph of the zigzagged structures, indicating the spacing of the corners. The radius of curvature of the corners was 2.5 $\mu \mathrm{m}$. To inhibit non-specific adhesion of cells to the surface due to protein adsorption from the cell culture medium, the sample surface was coated with $15 \mathrm{~nm}$ of tetraglyme by plasma polymerization [Salim 2007]. Microwires were fabricated on Si chips and $100 \mathrm{~nm}$ thick $\mathrm{Si}_{3} \mathrm{~N}_{4}$ membranes for confocal fluorescence and x-ray imaging, respectively.

Magnetic transmission X-ray microscopy (M-TXM) was performed at beamline 6.1.2 at the Advanced Light Source in Berkeley, CA using a photon energy of $853 \mathrm{eV}\left(\mathrm{Ni} \mathrm{L}_{3}-\right.$ absorption edge). Magnetic contrast was achieved at a spatial resolution of around $25 \mathrm{~nm}$ via X-ray magnetic circular dichroism (X-MCD) by holding the sample at an angle of $60^{\circ}$ to the X-ray optical axis. Using opposite circular polarizations or using an image taken at saturation as a reference image [Bryan 2008], magnetic contrast was increased further by digitally dividing two raw images. Full details of the experimental arrangement of the microscope can be found elsewhere [Fischer 2006, Fischer 2008].

\section{FIGURE 1HERE}

RN22 rat Schwann cells were cultured under standard conditions $\left(37^{\circ} \mathrm{C}, 95 \%\right.$ air $/ 5 \% \mathrm{CO}_{2}$ humidified atmosphere) in Dulbecco's Modified Eagle's Medium (DMEM) + 10\% (v/v) FCS, 1\% penicillin/streptomycin, 1\% glutamine, and $0.25 \%$ amphotericin. 18 hours before the experiment, the culture medium was exchanged for medium mixed with a magnetic bead stock (360 nm diameter Spherotech Fluorescent Nile Red Carboxyl Magnetic Particles) at a ratio of $10 \mathrm{ml}$ medium:10 $\mu$ bead stock. Prior to imaging, cells were stained following a standard protocol for CellTracker Red (Invitrogen): cells were incubated for 45 minutes in a $100 \mu \mathrm{g}: 14.6 \mu \mathrm{l}: 146 \mathrm{ml}$ solution of CellTracker Red:dimethyl sulfoxide (DMSO):serumfree medium, then incubated for a further 30 minutes in fresh serum-free medium. Cells were lifted-off, washed in serumfree medium and re-suspended in phosphate-buffered saline (PBS) with around $4 \times 10^{5}$ cells $/ \mathrm{ml}$.

A Zeiss LSM510 META upright confocal microscope employing long-range water-dipping objective lenses was used to demonstrate that the zigzagged structure operated as a switchable trap. Using an excitation wavelength of $543 \mathrm{~nm}$ and an emission wavelength of $600-650 \mathrm{~nm}$, fluorescently labeled cells and beads were imaged simultaneously. Before introducing the cells, a $6.4 \mathrm{kA} / \mathrm{m}$ field was applied along the $x$ axis, so that domain walls were formed at remanence. The cells were allowed to settle onto the surface for 45 minutes. They were then washed in a pulsatile flow (amplitude 82 $\mu \mathrm{l} / \mathrm{pulse}$ ) to remove cells not anchored to the surface, progressively increasing the average flow velocity to $0.5 \mu \mathrm{m} / \mathrm{s}$ (50 pulses/min) over a 30 minute period. With the flow turned off, a $6.4 \mathrm{kA} / \mathrm{m}$ magnetic field was applied in the $y$-direction to remove the domain walls and switch the trapping function of the microwires. Finally, the sample was washed for 10 minutes in a $0.5 \mu \mathrm{m} / \mathrm{s}$ pulsatile flow to remove cells no longer attached to the sample surface.

\section{TRAP DESIGNS}

Fig. 2 shows M-TXM images and micromagnetic calculations of representative magnetization configurations of SMW, PMW and CMW, together with the modeled force profile $15 \mathrm{~nm}$ above the surface of each structure. For each structure there is good agreement between the M-TXM and modeled magnetization configurations, suggesting that the model is representative of the experimental system. SMW (Fig. $2 a$ ) is similar to previous designs used to trap cells [Tanase 2005] in that it is single domain and has suppressed closure domain structure. The structure produces a $78 \mathrm{pN}$ maximum trapping force. Experimentally, forces of around $100 \mathrm{pN}$ have been shown to capture beads [Mirowski 2004], indicating that the model predicts an appropriate order of magnitude for trapping beads. However, the trapping force cannot be switched off to release cells as the wire end will always emit a field.

In PMW, the net force is dependent on the wire magnetization configuration. By applying a field along one wire axis, either the head-to-tail (Fig. 2b) or the tail-to-tail configuration (Fig. 2c) can be accessed. The head-to-head configuration (not shown) generates forces identical to the tailto-tail configuration. The proximity of the wire ends changes the closure domain structure from that found in SMW, modifying the field produced. Furthermore, the distribution of the field sources means that the field is not simply doubled or cancelled out and the force profiles are not a superposition of two SMW force profiles Hence, the maximum force resultant 
from the tail-to-tail configuration $(122 \mathrm{pN})$ is not quite double that from SMW, and the maximum force from the head-to-tail configuration is almost as strong as SMW (76 pN).

\section{FIGURE 2 HERE}

Contrast between the ON (high trapping force) and OFF (low trapping force) states is higher in continuous wires, such as CMW. Domain walls formed in the corner after saturating along the $x$-axis can be removed by fields applied along the $y$ axis (Figs. 2d-e). The corner causes the domain wall to have an asymmetric vortex structure (Fig. 2d), where one section of the vortex is more prominent than the others. Vortex asymmetry was not observed in narrow wires [Bryan 2008], as these wires had higher shape anisotropy than the present study. The structure of the asymmetric vortex wall results in a more complicated force profile and lower field than seen above SMW or PMW. The maximum trapping force from the wall is $67 \mathrm{pN}$ (Fig. 2d), similar to SMW. With no domain wall present (Fig. 2e), the maximum trapping force was only 0.02 $\mathrm{pN}$, comparable with the numerical noise in the model.

\section{DEMONSTRATING CELL TRAPPING}

The large difference in the calculated force from the two magnetic states indicates that corners could be used to trap and then release cells containing superparamagnetic beads. To demonstrate this, cells labeled with beads were deposited onto sets of zigzagged microwires that had been magnetized with domain walls present (ON state). Fig. 3a shows the cells (dark globules) above the microwires after falling out of suspension. The superparamagnetic beads appear as small, bright areas. Not all the beads have been endocytosed and some occupy trapping sites instead of cells. The cells assembled individually on the sample surface, although a small degree of clumping can be seen in Fig. 3a, possibly because bonding between cells is stronger than the cell-surface adhesion. Under a pulsatile flow, unanchored cells migrated across the sample surface at a velocity of $0.5 \mu \mathrm{m} / \mathrm{s}$. Using this as the flow velocity and approximating the Schwann cells as $10 \mu \mathrm{m}$ diameter spheres, the drag force on the trapped cells can be estimated as $0.05 \mathrm{pN}$. After 10 minutes at this flow velocity, the majority of cells remaining on the sample surface were positioned above the corners of the microwires (Fig. $3 b$ and c). In general, the cells were largely organized along the axis defined by wire sections connecting the closest corners, indicating that the design may be used to pattern cells. As a measure of the efficiency of the trapping, the number of cells or beads trapped above a corner was compared with the total number of cells or beads over an area of $250 \mu^{2}$. Around $65 \%$ of the cells/beads present were trapped above a corner, while a further $31 \%$ were anchored to trapped cells, sometimes via other cells. The remaining $4 \%$ had no connection to the trapping sites. The pattern from cells that are directly trapped by the domain walls is transferred to the cells anchored to them. These indirectly patterned cells are also able the transfer the pattern to cells attached to them, although the fidelity of the pattern transfer progressively degrades as the number of cells in the aggregate increases. To demonstrate that the patterning of cells is not due to surface defects, the trap was set to the OFF state (no domain walls) and the pulsatile flow reapplied. 94\% of cells were washed away (Fig. 3d), indicating that the cell anchoring seen in Fig. $3 \mathrm{~b}$ was indeed due to magnetic interactions between the beads within the cells and the domain walls in the corners.

\section{FIGURE 3 HERE}

Cellular organization is a fundamental requirement for controlled tissue growth. By aligning Schwann cells, we open up the possibility of studying the effect of cellular organization on the glial-neuronal interaction, which is essential for reinnervation. In principle, the technique could be applied to any cell culture model where cellular organization affects intercellular interactions. Alternatively, as cells can be moved away from trapping positions, the technique could potentially form part of a microfluidic platform in which cells are moved between different test points [Vieira 2009].

\section{CONCLUSION}

Lithographed micromagnets were designed to allow Schwann cells incorporating superparamagnetic beads to be organized on a magnetically patterned surface by exploiting the attraction between the beads and magnetic fields from the micromagnets. Subsequently, the magnetization of the pattern was changed, allowing the cells to be removed from the surface. The technique could be applied to any cell type, enabling studies into the effects of cellular organization on cell growth and co-culture development.

\section{ACKNOWLEDGMENT}

This work was supported by the BBSRC (grant BB/F015844/1 ), the EPSRC (grant EP/D056683/1) and by the Basic Energy Sciences, of the U.S. Department of Energy under Contract No. DE-AC02-05CH11231.

DAA acknowledges an Advanced Research Fellowship from the EPSRC (grant GR/T02942/01).

\section{REFERENCES}

Allwood DA, Schrefl T, Hrkac G., Hughes IG and Adams CS (2006). Appl. Phys. Lett., vol. 89, pp. 0141021.

Allwood DA, et al. (2005). Science, vol. 309, pp. 1688-1692.

Bryan MT, Fry PW, Fischer PJ and Allwood DA (2008). J. Appl. Phys., vol. 103, pp. 07D909.

Conroy RS, Zabow G, Moreland J and Koretsky AP (2008). Appl. Phys. Lett., vol. 93, pp. 203901.

Desai JP, Pillarisetti A and Brooks AD (2007). Annu. Rev. Biomed. Eng., vol. 9, pp. 35-53.

Fischer P (2008). IEEE Trans. Magn., vol. 44, pp. 1900.

Fischer P, et al. (2006). Materials Today, vol. 9, pp. 26.

Fredkin DR and Koehler TR (1990). IEEE Trans. Magn., vol. 26, pp. 415-417. 
Inglis DW, Riehn R, Sturm JC and Austin RH (2006). J. Appl. Phys., vol. 99, pp. 08K101.

Mirowski E, Moreland J, Russek S, Donahue M and Hsieh KW (2007). J. Magn. Magn. Mater., vol. 311, pp. 401-404.

Mirowski E, Moreland J, Russek SE and Donahue MJ (2004). Appl. Phys. Lett., vol. 84, pp. 1786-1788.

Parkin SSP, Hayashi M and Thomas L (2008). Science, vol. 320, pp. 190-194.

Salim M, et al. (2007). Lab Chip, vol. 7, pp. 523-525.

Schrefl T, Schabes ME, Suess D and Stehno M (2004). IEEE Trans. Magn., vol. 40, pp. 2341-2343.

Sun T, et al. (2008). Biotechnol. Bioeng., vol. 99, pp. 1250-1260.

Tanase M, et al. (2005). Lab Chip, vol. 5, pp. 598-605.

Vavassori P, et al. (2009), arXiv:0903.3516v1.

Vieira G, et al. (2009). Phys. Rev. Lett., vol. 103, pp. 128101. a)

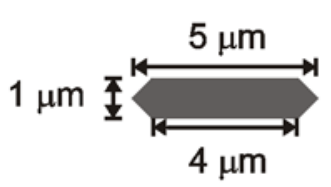

c)

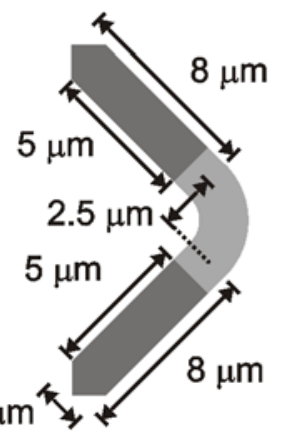

b) $1 \mu \mathrm{m}$

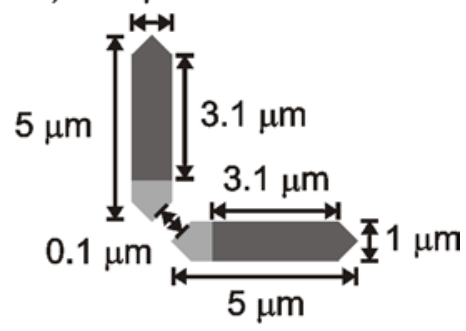

d)

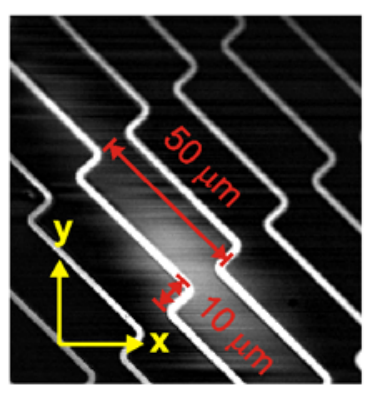

Fig. 1: Schematic diagrams of the structures modelled: (a) single microwire, SMW, (b) perpendicular microwires, PMW, and (c) curved microwire, CMW. In each wire, the taper length is half the width. The lighter areas indicate a finely meshed region. (a) Confocal micrograph of experimental microwires, showing the corner-to-corner spacing and the coordinate axes used. a)

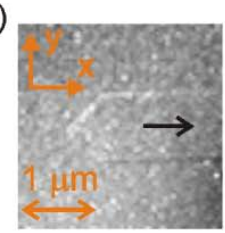

b)

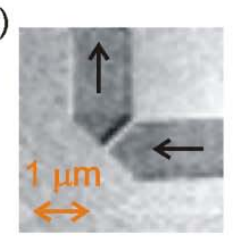

c)

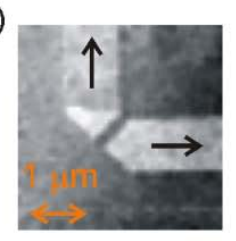

d)

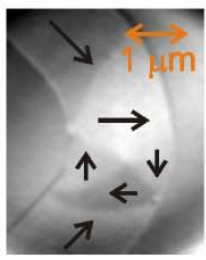

e)

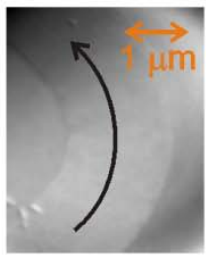

$\mathrm{M}_{\mathrm{x}}$

$\begin{array}{lll}-1 & 0 & 1\end{array}$

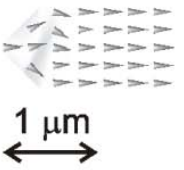

$\stackrel{1 \mu \mathrm{m}}{\longleftrightarrow}$
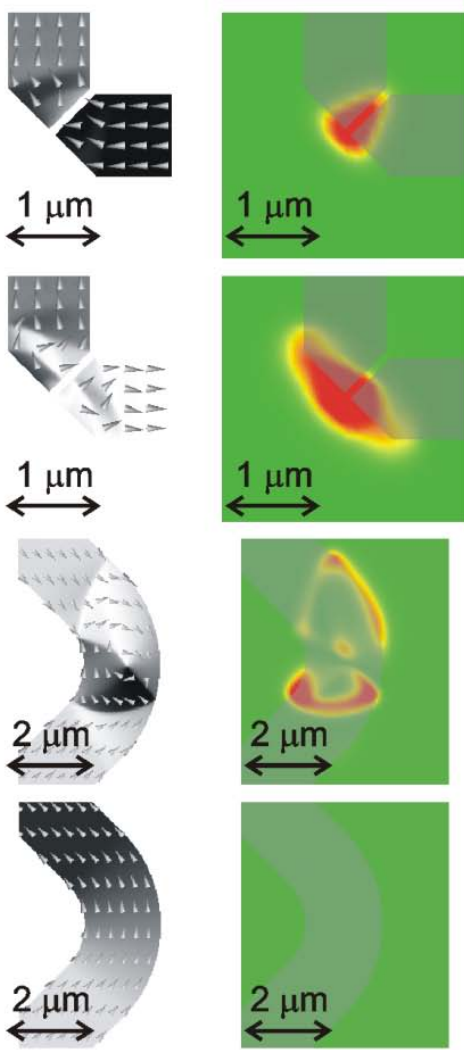

$F(p N)$
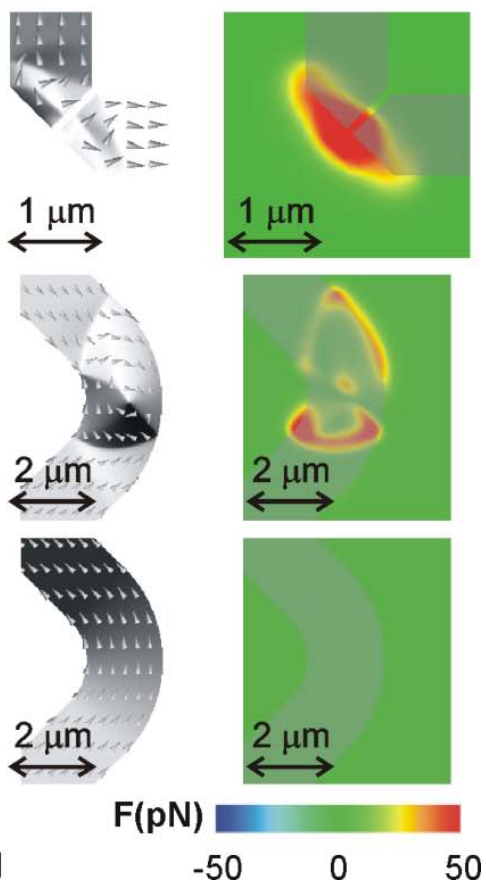

$-50$

0 50

Fig. 2: M-TXM images and the micromagnetically calculated magnetization state, with the corresponding force on a bead $15 \mathrm{~nm}$ above the surface for sections of (a) SMW, (b) PMW in the head-totail configuration, (c) PMW in the tail-to-tail configuration, (d) CMW (zigzagged structure) with a domain wall and (e) CMW without a domain wall. Arrows indicate magnetization direction. All structures are oriented to the coordinate axes defined in (a). 

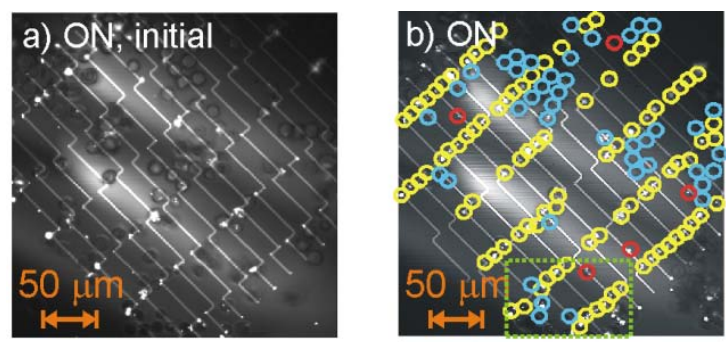

c) ON, detail
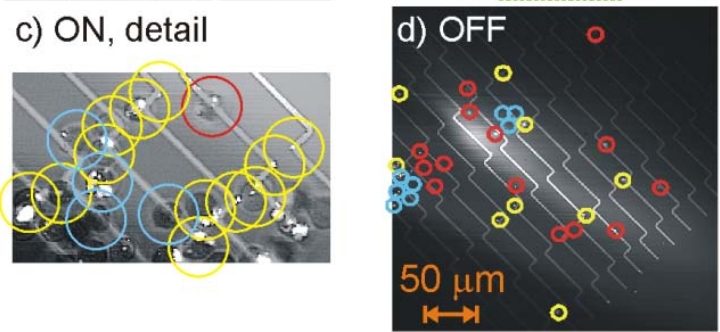

- Trapped on corner Indirectly trapped

Not

Fig. 3: Confocal micrographs of the cell distribution above CMW. (a) Before and (b) after applying a pulsatile flow with the trap turned on (domain wall present). (c) Enlargement of the dotted area in (b). (d) After the trap was turned off (domain walls removed) and the flow reapplied. The rings highlight the trapping state of the cells and beads. 